Cahiers d'études italiennes

\title{
Désir et répulsion : Aracœli ou le miroir paradoxal
}

\section{Agnès Morini}

\section{OpenEdition \\ Journals}

Édition électronique

URL : http://journals.openedition.org/cei/826

DOI : $10.4000 /$ cei.826

ISSN : 2260-779X

\section{Éditeur}

UGA Éditions/Université Grenoble Alpes

Édition imprimée

Date de publication : 15 septembre 2006

Pagination : 151-161

ISBN : 978-2-84310-086-4

ISSN : $1770-9571$

Référence électronique

Agnès Morini, « Désir et répulsion : Aracœli ou le miroir paradoxal », Cahiers d'études italiennes [En ligne], 5 | 2006, mis en ligne le 15 mars 2008, consulté le 28 mars 2021. URL : http:// journals.openedition.org/cei/826 ; DOI : https://doi.org/10.4000/cei.826 


\title{
DÉSIR ET RÉPULSION
}

\author{
ARACELI OU LE MIROIR PARADOXAL
}

\author{
Agnès Morini \\ Université Jean Monnet - Saint-Étienne
}

La fable de Manuele, le protagoniste du dernier roman d'Elsa Morante ${ }^{1}$, est, comme toutes les fables, cruelle, féroce même, mais à la différence des autres, elle est sans morale, elle ment et elle ensorcelle pour ne rien révéler d'autre que son mensonge; au contraire d'une initiation ou d'une catharsis, elle est un piège inéluctablement refermé, une impasse: "C'era una volta uno specchio dove io, mirandomi, potevo innamorarmi di me stesso : erano i tuoi occhi, Aracoeli, che m'incoronavano re di bellezza nelle loro piccole pozze incantate» (p. 107).

Un jour - celui où on lui met sur le nez ses premières lunettes -, Manuele, littéralement contraint d'ouvrir les yeux, lit dans ceux de sa mère le premier signe d'une disgrâce définitive: «Per la prima volta nella [sua] vita, essa [lo] vedeva brutto ${ }^{2}$. C'est alors et de cette manière que Manuele sort brutalement des «limbes ${ }^{3}$ ", chassé à vie du "jardin magique» ou «jardin d'amour» par celle dont le nom promet pourtant exactement l'inverse (p. 123). Il ne se sentira plus jamais beau: «Presto mi fu chiaro ch'io non posso piacere a nessuno, come non piaccio a me stesso» (p. 14).

1. Notre édition de référence est l'édition princeps, Turin, Einaudi, 1982.

2. p. 174; «Senz'altro, allora essa dovette rendersi conto, invincibilmente, che suo figlio, crescendo, s'imbruttiva; e che accusarne soltanto gli occhiali sarebbe [...] un falso alibi. In verità, sullo stampo primitivo del mio viso, che tanto la innamorava, già cominciava a lavorare quel pollice oscuro e maligno che doveva deformarlo senza rimedio, per la mia eterna disgrazia» (p. 175). Voir aussi p. 172 et 283.

3. Nous faisons allusion au dernier vers de la dédicace de L'isola di Arturo, «fuori del limbo non v’è Eliso". 
Pire encore, il n'aura plus de son corps d'autre image que de répulsion. Dans un miroir, celui sans concession d'une médiocre chambre d'hôtel cette fois, son corps qui apparaît ne lui inspire qu'une verbalisation dépréciative et les sentiments corollaires de dégoût de soi ${ }^{4}$ et d' "antipathie", pour reprendre un terme qui figure dans les pages auxquelles je fais allusion (p. 106-107). Ne se reconnaissant pas dans l'enveloppe humiliante de sa chair, Manuele cherche au fond du miroir de ses propres yeux, cet autre lui-même, «le seul, le vrai», cet enfant qu'il fut aux temps aveugles d'avant le désamour d'Aracoeli, et dont il ne parvient pas à faire le deuil ${ }^{5}$. Mais ses pupilles ne reflètent qu'une "petite ombre diluée (presque naufragée)", mirage fabriqué par les yeux maternels, qu'il poursuit, "canuto Narciso ", derrière tous les "narcisi imberbi" auprès desquels il mendie des miettes d'amour - «mi umiliavo in implorazioni mi prostravo gemevo» (p. 107)-. Et si nous employons le verbe "mendier», c'est que tout le comportement amoureux de Manuele reproduit celui de l'enfant de cinq ans qui se découvre laid dans les yeux de sa mère - «veggenza lacerante»et essaie d'effacer des lèvres d'Aracœli un sourire forcé par son propre sourire "di rimedio", "di elemosina", "d'ansia interrogativa e di seduzione», appelant un baiser ou une caresse qui, en quelque sorte, le ré-aveugle (p. 174-175). Adulte suppliant et pleurnicheur, Manuele n'obtient généralement de ses amants que d'être objet de leurs «dileggi, ribrezzi, ricatti, percosse e linciaggi» (p.107). C'est ce qu'illustre sa pitoyable aventure avec Mariuccio, sur le mode d'un «je t'aime moi non plus» dans lequel lui échoit le rôle du mauvais stratège:

In un trattato sulla strategia amorosa avevo letto che i grandi seduttori da ultimo ricorrono alla tattica dell'assenza per trasformare un'ordinaria avversione in una nostalgia straordinaria. Ma alla mia assenza, invece, corrispose solo una calma spettrale. (p.49)

Un calme après une tempête de rebuffades et d'insultes parmi lesquelles ce «Non lo vuoi capire che mi fai schifo?» n'est peut-être pas la pire (p.48). La monstrueuse image que Mariuccio renvoie à Manuele n'est du reste qu'une variante verbale et injurieuse de cette «forma reale» que le miroir d'un petit hôtel d'Almeria "[gli] butta in faccia, senza cerimonie", de nombreuses années plus tard (p. 107): «Sempre per la stessa domanda, o millanteria, o pretesa [celle d'être aimé, bien sûr], ci si consegna alla strage e alla croce e al sadismo e all'algolagnia e al saccheggio e alle macerie» (p. 107).

4. «E chi non si schiferebbe di questa scimmia, quando me ne schifo io medesimo?» (p. 107).

5. Voir p. 106-107. 
Mais, de station en station d'un interminable chemin de croix, la passion de Manuele ne rachète rien ni personne: «La mia domanda assillante ormai si legava inesorabilmente [...] al tema della colpa e della vergogna. Ho rinunciato, alla fine, a ogni domanda; ma la colpa e la vergogna perdurano ${ }^{6}$ ".

Ange déchu, Manuele vérifie dans sa chair le décret divin «Vi vergognerete della vostra nudità », auquel on negligea d'ajouter : "E avrete bisogno di carezze fino all'ultimo vostro giorno" (p. 108). Toutefois la loi est injuste, qui permet aux uns de séduire, à d'autres de n'être que les pantins d'un jeu de massacre - "pupazzo [...] disarmato e sfasciato, una sagoma da tiro a segno» (p. 108-109):

Favoriti, infatti, fra i mortali, sono i giovani belli, che possono offrire senza vergogna alle carezze la propria carne radiosa. E riscattati coloro che, almeno, possono offrire qualche altro sfoggio, da farsene piacenti : esempio i campioni; i taumaturghi, i poeti. Ma io? Da offrire non ho niente ${ }^{7}$.

Tel est le drame humain selon Manuele: «Ogni creatura, sulla terra, si offre. Patetica, ingenua, si offre: "sono nato! eccomi qua [...]. Vi piaccio? mi volete?" [...] "vi paio bello?" ; " unica perpetua domanda di ogni vivente agli altri viventi» (p. 107-108):

E ciascuno, allora si dà a esibire le proprie bellezze: donde si spiegano le nostre vanità disperate. [...]. Orfani e mai svezzati, tutti i viventi si propongono, come gente di marciapiede, a un segno altrui d'amore. (p. 107)

Inutile de multiplier les exemples de cette nature, qui sont légion. On aura compris à ces quelques rappels que la logique du désir et de la séduction induite par le discours de Manuele est une logique de destruction. Et dans ce roman, nul n'y échappe.

Aracœli moins que les autres. Somme de toutes les femmes et mères des romans d'Elsa Morante, elle est l'illustration d'une nature à deux dimen-

6. p.14. Le thème de la condamnation collective du paria est introduit dès cette page: "Quando mi succede di trovarmi fra una folla, io mi sento l'oggetto designato per un linciaggio. Il giudizio innumerevole del Collettivo punta le sue pupille omicide addosso al mio corpo " (p. 15).

7. p. 108. On se souvient de l'Elisa de Menzogna e sortilegio, qui tend à se dénigrer au plan physique (elle évoque une "antica avversione per la [...] propria figura" qui rappelle très directement l'autodévaluation de Manuele - p. 12 dans l'édition Turin, Einaudi, 1975, "Gli struzzi» 72), à faussement s'apitoyer sur son sort, tentant ainsi d'attirer sur elle la compassion, l'attention donc, de son lecteur, de l'impliquer dans une relation directe qui lui permette de transférer sur lui la frustration irrésolue de l'amour de ses parents. Elisa, faute de séduire son lecteur en imposant une beauté qu'elle dit ne pas avoir, l'attire dans les filets du récit qu'elle tisse. Sa coquetterie, sociale en somme, est celle de la conteuse qui s'exhibe pour être reconnue comme essere scrivente. Elle est, pour reprendre la classification de Manuele, du côté des poètes, des «riscattati». 
sions inconciliables entre elles: la maternité et la sexualité. Dans sa dimension maternelle, Aracœeli se caractérise par trois traits: la permanence de l'enfance, la référence mariale et le statut royal (ragazza, Vergine et regina sont de fait les qualificatifs récurrents et emblématiques qui la définissent). «Macchina idiota», nous dit Manuele, "serva ignara» de ses propres mécanismes, «il [...] corpo, immune da ogni tentazione erotica», "senza languore né civetteria» (p.104), elle est porteuse de cette "attente inconsciente de la semence» pour remplacer ses poupées per "una bambola di carne viva»: "Esplorando fra le nebbie delle tue chimere bambinesche, forse si scoprirebbe che tu, allora, contavi su una fecondazione «senza peccato» comme quelle della Virgen» (p. 103-104).

Il n'y a encore chez cette Aracoeli-ci qu'une sorte de coquetterie instinctive, génétique, mise en œuvre pour accéder à la maternité. La chambre conjugale reste le lieu d'une «investitura sacra»(p.136). Mais quand la mère n'est plus - ce que marque symboliquement la mort de la petite Carina - , la femme, érotisée, se contorsionne dans le ballet nymphomaniaque, bestial et obscène d'une sorte de pestiférée ${ }^{8}$. La demande d'amour d'Aracœli est alors proférée par une voix définitivement "laida e plorante di femmina» (p. 239) et un appel du corps sans ambigüité:

$\mathrm{Al}$ suo passo ondulante, le curve del suo corpo si proponevano, ingenue e sfrontate [...]. E le voci di lode alla sua bellezza, le occhiate parlanti, parevano toccare fisicamente la sua epidermide, come lingue o dita, o mordere la sua pelle fino agli strati profondi, facendola trasalire. Di quando in quando, [...] si lasciava in pose che una volta le avrebbero fatto vergogna. Si tendeva sul busto [...]. Accavallava le gambe con una incuranza volgare [...]. E se da un tavolo vicino un uomo prendeva a fissarla [...] essa ne risentiva una emozione ansiosa, udibile e visibile nei suoi respiri. (p. 236)

Et plus elle s'offre, plus elle se donne, plus Aracœli se dégrade, au propre comme au figuré: les dernières descriptions du personnage multiplient les mots du gâchis, de l'avilissement et de la déchéance. Par ailleurs, plus elle se détruit, plus Aracœli corrompt ceux qui l'entourent. Parmi eux, Daniele, l'ordonnance de son mari, un jeune marin qui avait conservé la fraîcheur limpide et confiante de l'enfance, mais qui, attiré dans le lit

8. L'image d'un mal bestial qui tenaille Aracoli - «occulte mandibole lavoravano con ferocia nel suo ventre» (p. 249) - s'accompagne de celle de la contamination - «lebbra nascosta» (p. 248) ou «morbo ossessivo» (p. 249). C'est la différence la plus grande entre Aracœeli et les personnages féminins des romans précédents d'E. Morante: Aracoli offre l'image d'une excroissance montrueuse de la volupté de l'Anna de Menzogna, comme si l'on était passé, du premier au dernier roman, de l'idylle de roman de gare à la pornographie. C. Garboli évoque, pour sa part, une "Madonna capovolta, sconsacrata, rovesciata" (Il gioco segreto. Nove immagini di Elsa Morante, Milan, Adelphi, 1995, «Piccola Biblioteca», p. 197). 
d'Aracœli, est métamorphosé par un «hurlement bestial» - «da scimmia o da gatto selvaggio ${ }^{9}-$, après lequel sa voix sera « irriconoscibile, stonata $e$ monca», puis toute "gemiti rantolanti» du jeune suicidaire qui a ingurgité un quelconque détergent à sa portée (p. 263). Quant à Eugenio, le père de Manuele, il est une présence solaire dans la première moitié du roman, nimbé d'un vague héroïsme, véritablement aimé des dieux (il s'appelle aussi Amedeo) et haussé à leur rang. Toutefois, s'il est dans un premier temps consacré par la "fede adorante d'Aracœli» (p. 183), il décrit ensuite avec cette dernière la courbe descendante d'une éloquente décrépitude: aux dernières pages du roman - qui lui sont presque entièrement consacrées -, sale, ivre, le corps déformé et malodorant, la peau maculée et sanguine, incapable de proférer autre chose qu'une série de mots informe "quasi idiota, finché gli si disfaceva in un impasto di suoni disarticolati e inservibili» (ibid.), Eugenio n'inspire à Manuele que «ribrezzo» (p. 321), "un cupo imbarazzo senza nessuna pietà » (p.323) et "una torva antipatia» (p.324). Aracoli, séductrice malade, jette sur le monde qui l'entoure la malédiction ravageuse du pourrissement et de la dissonance.

Il est alors logique que Manuele, éconduit et donc désenchanté (dans tous les sens du terme), nous donne du monde qui l'entoure une vision (et un point de vue narratif) pour le moins déformée, et ses défauts visuels y contribuent certes largement - il est à la fois myope, presbyte et astigmate: "Via via che il mondo [...] s'andava offuscando e storcendo alla mia vista menomata, nel medesimo tempo l'acqua delle mie iridi s'intorbidava» (p. 175).

On remarque ici un nouvel effet de miroir: la vue défectueuse du personnage "trouble» le monde, et le monde "trouble» sa vue. Dès qu'il enfourche ses premières lunettes, Manuele enfant découvre un "mai veduto spettacolo d'orrore», un monde dont il saisit la clarté et le relief comme "un'unica violenza proteiforme" (ibid.). Il aura beau essayer d'ôter ses lunettes pour en repousser la laideur et en même temps reconquérir sa mère, rien n'y fera. Adulte, il gardera d'ailleurs l'habitude d'enlever et remettre ses lunettes dans l'espoir tout aussi vain de démêler ses

9. P. 260. Et par delà la mort, dans une hallucination de son fils, elle apparaît encore, silhouette dansante, mais «il suo ballo è una sconcia parodia dell'adescamento puttanesco. Via via [...] essa si spoglia $[\ldots]$ e le sue nudità scoperte provocano a vederle la stessa vergogna misera e irremediabile che fa torcere lo sguardo dai corpi degli animali scuoiati» (p. 130). 
fausses visions des vraies ${ }^{10}$. Bref, quoi qu'il fasse, Manuele appréhende le monde dans la difformité du gigantisme, du rétrécissement, de contorsions et d'effets lumineux de tous ordres, qui excluent toute harmonie. Il s'ajoute à cela toutes sortes de brouillages sonores qu'accentuent encore les effets chimiques de l'alcool, des drogues ou des narcotiques ingérés par le personnage: ses divagations visuelles et auditives deviennent pour le coup proprement «stupéfiantes». Or, il ne s'agit pas tant, pour Manuele, de fuir le monde, fût-ce dans le sommeil artificiel - «Ho imparato a praticare il sonno come sperpero, sciopero e sabotaggio. E questi ultimi anni, io li ho spezzati e disfatti, disertando la fabbrica del tempo [...]. Alla mia assenza, gli orologi del mondo saltavano, e le giornate si fogliavano in disordine...» (p. 51) - que de se laisser repousser par lui ; il s'agit en fait de se soumettre à un bouleversement de l'espace et du temps, de contribuer à subvertir l'ordre du monde (le pôle répulsif), pour accomplir le voyage à rebours vers Aracœeli (son pôle d'attraction), comme le soulignent ces deux passages si emblématiques:

Ribaltare il sistema del tempo. Il futuro all'indietro, il passato viene incontro. E in questa anarchia falotica, di là dalle croci dei giorni, c'è lei che mi aspetta ${ }^{11}$.

Verso El Almendral, i tempi si riducono a un unico punto sfavillante: uno specchio, dentro il quale precipitano tutti i soli e le lune. (p. 22)

Ce point spatio-temporel aimanté, ce Nord de la boussole interne de Manuele, pourrait-on dire ${ }^{12}$, trouve par ailleurs un équivalent phonique dans la voix d'Aracœeli, "proprio la voce fisica di lei, col sapore tenero di gola e di saliva» (p. 10), qui semble l'aspirer vers El Almendral ${ }^{13}$. Et Manuele de nous expliquer que ces manifestations ne sont pas une " trans-

10. «Io mi metto, e mi ritolgo, e mi rimetto gli occhiali per ritrovare sempre il solito sfacelo di sassi e rupi» (p. 131); "gli oggetti comuni mi si trasmutano in sagome stravaganti e indecifrabili [...] Né sempre gli occhiali bastano a soccorrermi» (p. 20).

11. P. 51. D'autant que Manuele affirme par ailleurs: «Io [...] sono tentato a inseguire la mia ragazza Aracœeli in tutte le direzioni dello spazio e del tempo, fuorché una a cui non credo: il futuro» (p.7), et: «Mi sono messo sulla strada [...] per andare alla ricerca di mia madre Aracoeli nella doppia direzione del passato e dello spazio" (p.9).

12. En se référant à ces réflexions: "Quel minimo punto [...] da ultimo era diventato l'unica stazione terrestre che indicasse una direzione al mio corpo disorientato", "portato dai [miei] sensi acuti, [io rifaccio] tutto il cammino all'indietro, verso il punto del principio (forse a una agnizione?)» (p.9). Du reste, ce «richiamo senza nessuna promessa, né speranza» (ibid.) qui attire Manuele vers sa préhistoire nous paraît une forme inversée de celui qui happe Ida et Useppe vers l'«atroce Paradis» auquel est destinée l'humanité (La storia, Turin, Einaudi, 1974, "Gli struzzi» 58, p. 337 à 340).

13. Il convient de rappeler que Manuele souhaite carrément être ré-ingugité par sa mère: «Ma tu, mamita, aiutami. Come fanno le gatte coi loro piccoli nati male, tu rimàngiami. Accogli la mia difformità nella tua voragine pietosa» (p. 109). 
cription abstraite de la mémoire» (p. 109), mais le fruit de ce qu'on pourrait qualifier de "mémoire organique», un sixième sens capable de voir, d'entendre et de sentir par delà les dimensions réelles, «in quella zona [in cui] si avvera [...] la resurrezione carnale dei morti» - on comprend du même coup que Manuele, en mal du corps de sa mère, réponde aux stimuli de ce cordon mnémonique (p. 10). Cependant cette mémoire-ci est une sirène, comme le révèle ce passage indispensable à notre propos:

In genere si suppone che, al momento della nascita, la nostra memoria sia un foglio bianco ; però non è escluso che, invece, ogni nuovo nato porti in sé la stampa di chi sa quali soggiorni anteriori, con altre nature e altre luci. Forse queste, agli esordi del suo soggiorno terrestre, interferiscono ancora, simili a una lente aberrante, nelle nuove apparenze quotidiane offerte alla sua retina. E allora il suo campo s'inonda di forme e colori favolosi, per via via ridursi, impallidendo nel tempo, alla povertà di una sinopia dopo lo strappo dell'affresco. Finché la memoria adulta (comunemente, almeno) provvede a dissipare fino all'ultima ombra di quel primario spettro luminoso. Considerandolo, a distanza, nient'altro che un effetto equivoco, falso e strumentale: il quale forse, con le sue fantasmagorie precarie, voleva consolarci della nascita, così come le visioni leggendarie dell'al di là vorrebbero consolarci della morte. (p.111)

Nous voudrions d'abord remarquer le recours, ici particulièrement suggestif, au langage de la physique optique; ensuite, la connotation paradisiaque du passé "prénatal» et consolateur dont les couleurs illumineraient la prime enfance; puis la coïncidence entre la sortie de l'enfance et la désillusion littérale: le "spectre lumineux" est un spectre, l'effet d'optique (de mémoire) est un effet. Et force est de déduire de ce propos que l'Aracœli des origines après laquelle court Manuele, et qui, selon cette conception, appartiendrait au royaume des "fantasmagories précaires", est ainsi renvoyée à sa fonction de fée Morgane et de mirage ${ }^{14}$. Et même le souvenir de l'Aracoli des cinq premières années de Manuele, celle de la maison des Quartieri Alti, pourrait bien n'être qu'une «reconstruction visionnaire» (l'expression est empruntée à Manuele, p. 11), une image repêchée dans les eaux du fleuve Restitution: " $\mathrm{E}$ in quest'altro fiume che si ribevono le memorie perdute; ma come accertarsi che le sue acque non siano drogate, e inquinate da presagi o seduzioni, fabulazioni o inganni?» (p.166). Manuele évoque du reste plusieurs fois des souvenirs apocryphes - « ricordi apocrifi» ou « rimembranze apocrife» ${ }^{15}$, que voici justi-

14. Dans le même registre, la mémoire est par ailleurs qualifiée de «brumosa necropoli» hantée de «fantasmi [...] larve minaccianti del passato. Miraggi negativi [...] larve o miraggi: vale a dire fumo, zero" (p. 165).

15. Respectivement p. 11 et 13. 
fiés. La mémoire ainsi conçue explique également les impressions de déjàvu qu'il éprouve et qu'il interprète comme autant de reflets entre plusieurs vies, de jeux de miroirs! Voilà pourquoi Manuele croit reconnaître son parcours vers El Almendral:

Chi sa in quale altra mia biogafia, già una volta mi sono avviato in questa direzione [...]. Può darsi che quell'altra mia biografia sia soltanto immaginaria, un riflesso effimero di questa; ma anche è possibile che questa odierna, invece, sia solo un riflesso dell'altra: la vera $[\ldots]$ questa esistenza mia presente in realtà non sarebbe che l'ultimo di una serie infinita di riflessi ingannevoli. L'unica vera mia esistenza starebbe alla sorgente, di là dagli innumerevoli specchi deformanti che me ne contraffanno la figura. (p. 139)

Cette source vers laquelle il doit remonter à la rencontre de sa «vraie» vie, c'est bien sûr Aracœli, celle dont l'image est restée prisonnière du miroir qui ornait le mur de sa chambre à sa naissance. Le voyage en avion de Milan à Almeria, est, nous dit Manuele, comme un pèlerinage vers le "Sanctuaire du Miracle». C'est en tout cas un voyage à travers le miroir «dentro il suo specchio dalla sontuosa cornice, [dove] mi precede la mia staffetta encantadora: lei! Aracœli! ${ }^{16}$.

Les mots Miroir, miracle et mirage, qui dérivent, on le sait, d'une même souche, définissent pléonastiquement le personnage d'Aracœli en le plaçant dans le champ d'une séduction paradoxale et vénéneuse, c'est-à-dire qui attire (les hommes et la mort avec) et qui repousse (Manuele et avec lui la vie). Ainsi, en ce qui concerne Manuele, on constate l'effet quasi mécanique de cette séduction-répulsion: Aracœli morte, l'abandon consommé en quelque sorte, Manuele reflue en proportion vers leur point de fusion originel, le lieu de sa "vraie» vie, comme il finit par le croire; la séduction de sa mère est donc tout à la fois vitale et létale ${ }^{17}$.

Nous voudrions à présent proposer d'envisager la thèse selon laquelle le miroir qui emprisonne cette Aracoli est au fond une métaphore du roman éponyme. Un roman envoûtant, mais d'une âpreté qui voudrait en signi-

16. P. 20. Pour la première évocation de ce miroir, cf. p. 10-11.

17. Et il nous semble que cela se produit aussi par ricochet, par personne interposée: souvenons-nous qu'Eugenio, détruit par Aracœeli, devenu pour son fils objet de répulsion, révèle en même temps ce dernier à l'amour pour son père - «mentre mi rivoltavo di schifo alla sua presenza, io forse ne ero preso disperatamente d'amore» (p.328). Par ailleurs, la répugnance de Manuele pour le sexe féminin illustre le même paradoxe. Laissé aux mains d'une prostituée, Manuele, entre dégoût et illusion d'amour, explique: «Era intoccabile dai miei sensi, non tanto [...] perché repulsiva, ma piuttosto perché sacra» (p. 85). 
fier l'impuissance voire la mort. Un roman qui nous attire dans les mailles enchevêtrées des délires de Manuele en même temps qu'il nous repousse vers ses «matrices", les romans d'avant la mort de Pasolini - sous le sceau de laquelle il est placé, comme on le sait -, d'avant la vieillesse, la maladie et la tentation suicidaire d'Elsa Morante. Un roman qui, comme le miroir de la chambre d'Aracoeli, garde les images des trois autres et donc nous y renvoie au détour de chaque page. Alors certes, la mémoire romanesque, comme celle de Manuele, spéculaire et trompeuse, hésite parfois entre fidélité et distorsions, mais nombreux, très nombreux sont, dans Aracaeli, les échos thématiques, structurels et rhétoriques des trois romans précédents, tous degrés de déformation compris. Il n'est évidemment pas question de dresser un inventaire de ces coïncidences. Quelques exemples particulièrement significatifs suffiraient d'ailleurs à étayer une interprétation qui mériterait de toute façon plus que ces quelques lignes. Nous en retiendrons un surtout, celui de l'instance narrative.

Souvenons-nous que l'Elisa de Menzogna e sortilegio, et Manuele, sont imaginés dans des contextes existentiels initiaux qui, s'ils présentent de nombreuses différences, ont tout de même en commun de mettre en scène deux "malades de leurs parents", empoisonnés, intoxiqués (ces mots parcourent les deux ouvres) par du mensonge (et peu importe la nature de celui-ci, en réalité). Elisa, qui aspire à la guérison et à la revanche sur les fantômes des siens, nous raconte leurs vies dans un réordonnancement discursif systématique, s'affirmant peu à peu dans la maîtrise redoutable et ensorcelante d'une poésie; elle élabore pour nous, ses complices explicitement désignés, un roman organiquement structuré en parties, chapitres, sous-chapitres, dans lequel elle tient presque tous les rôles - de personnage, de narratrice, de «secrétaire" inspirée, d'écrivain. Aracoeli en revanche, nous propose une focalisation narcissiquement interne, presque asphyxiante ${ }^{18}$. Replié dans les volutes du cerveau détraqué d'un narrateur divisé entre un «vrai soi-même» et d'autres moins vrais, entre lui-enfant et lui-adulte, dans et en dehors de la réalité, ne se réalisant ni dans le féminin ni dans le masculin, bref constamment «réfléchi», dessaisi d'une identité stable - insaisissable ${ }^{19}$-, le récit, à l'inverse de la reconstruction métho-

18. Le rôle narratif de l'Arturo de L'isola est de toute évidence d'une complexité bien inférieure à celui d'Elisa, plus léger et limpide, au final. La Storia, ensuite, entre texte, paratexte, chapitres chronologiquement disposés, garde une ossature structurée, mais confie le récit à l'omniscience d'une voix extérieure avec, il est vrai, maints relais qui donnent souvent l'impression d'un roman choral, polyphonique.

19. Pour une forme aboutie et extrême de la schizé d'Elisa, en quelque sorte. Par ailleurs, alors que le narrateur de La Storia est positivement tout le monde, celui d'Aracali l'est négativement: il n'est personne. 
dique du familienroman d'Elisa, se présente comme une suite non orchestrée de fragments, discontinu, magmatique, conforme à l'incurable "natura scissa» de Manuele (p. 113), conforme aussi à son désir de revenir au temps informe d'avant sa naissance. Il n'est ni canalisé, ni dénoté, exempt de toute médiation métatextuelle, dépourvu du secours d'une voix unitaire et unifiante qui puisse réordonner la réalité dans la réalité romanesque $^{20}$. Manuele a perdu la distance démiurgique qui instituait Elisa en être narrante-scrivente: il ne crée pas, ni ne re-crée - la création du récit reste jalousement auctoriale, et sa recréation nous est totalement confiée $^{21}$ (on voit d'ailleurs poindre avec cette remarque un des arguments qui a pu faire parler de négation du récit dans Aracali). De ce point de vue, Aracali est une image totalement renversée de Menzogna e sortilegio. Mais bien sûr, nous aurions tout aussi bien pu rappeler comment Aracœli perpétue le mythe de l'enfance non pas comme le temps et le lieu d'un bonheur sans failles - il est facile de recenser les situations de solitude et de frustrations affectives que connaissent les enfants morantiens - , mais comme condition d'une grâce interdite aux adultes; comment il réaffirme la fonction tragiquement contaminatrice de cette pestilence qu'est l'histoire; comment en tuant l'Aracoeli «divine barbare» et avec elle toutes les Anna, Nunziata et Ida qui l'ont précédée, il ne réussit qu'à les sacraliser par le martyre de Manuele; et ainsi de suite. À l'inverse, d'aucuns se sont ingéniés à voir dans cet ultime roman d'Elsa Morante, celui de la négation $^{22}$, d'une amère palinodie ${ }^{23}$, celui du meurtre des autres, pour reprendre ici l'hypothèse de Cesare Garboli :

Aracoeli, est une parodie des romans morantiens vieille manière [...] c'est un roman bouleversé, brisé et au fond objet de dérision; les morceaux de verre qui le composent reflètent l'épave d'un ancien univers [...]. La parodie est un autodafé; une exhibition obscène; le geste par lequel ce que nous avons aimé le plus est déchiré. Aracali est ce geste $^{24}$.

20. Dans un article intitulé "Aracali: morte di Narciso", A.M. Zampolini observe fort justement que: "In Aracceli la narrazione ha perso la sua forma dinamica strutturante di uni-versum e gli elementi della storia ricadono nella più aspra confusione di tempi » (in Letture di Elsa Morante, ouvrage collectif, Turin, Rosenberg \& Sellier, 1987, "Quaderni/Gruppo La Luna», p. 186).

21. D'ailleurs Manuele nous exclut et nous met en garde: "Questa sorta di monologo sregolato, che vado qui recitando a me stesso, io l'ho imbastito, fino dall'esordio, coi fili dell'equivoco e dell'impostura" (p. 24).

22. Nous faisons allusion à G. Ferroni, qui définit Aracoli comme « una negazione definitiva di ogni esperienza» dans lequel E. Morante aurait voulu «bruciare per sempre la sua vita, la sua passione, la sua letteratura" (in Storia della letteratura italiana, Turin, Einaudi, 1991, Vol. IV, p. 561).

23. G. Rosa, Cattedrali di carta. Elsa Morante romanziere, Milano, Il Saggiatore, 1995, p. 293.

24. [Araceli è una parodia dei romanzi morantiani vecchia maniera [...] è un romanzo rotto, 
Appliquant ces jugements à l'espace diégétique ouvert par les pensées mortiferes de Manuele, on pourrait de fait y souscrire - et ils ne sont en outre pas forcément incompatibles avec notre propos sur certaines permanences thématiques ou idéologiques dans ce roman. Pourtant, si l'on considère cette fois le style de ce dernier roman, raffiné, travaillé, qui reproduit des oscillations entre une ligne haute et une ligne basse auxquelles les trois précédents nous avaient déjà habitués ${ }^{25}$, qui bouscule une fois encore les conventions d'équivalences sociales du langage, s'il l'on considère son hybridation générique aussi (et nous ne nous attarderons pas à démontrer qu'il reste dans Aracoli d'évidentes traces de fable, des traits parodiques, des chansons, des pages hautement lyriques, et même le dessin du talisman d'Aracœli!), n'est-il pas évident qu'Elsa Morante, pas plus qu'elle n'a tué le genre avec Menzogna e sortilegio ne parvient à le faire avec Aracoeli ${ }^{26}$ ?

Au miroir d'Aracoeli, c'est encore l'image de la poésie totale inventée par Morante qu'on peut voir, et elle n'a rien perdu de sa puissance émotive, de sa force onirique (fût-ce sous la forme maléfique du cauchemar) et... oui, en dépit de ses tentatives nihilistes, de son pouvoir de séduction.

Secondo certi negromanti, gli specchi sarebbero delle voragini senza fondo, che inghiottono, per non consumarle mai, le luci del passato (e forse anche del futuro ${ }^{27}$.

sconvolto, frantumato e in fondo deriso ; i pezzi di vetro che lo compongono riflettono i rottami di un antico universo [...]. La parodia è un autodafé; un'esibizione oscena; il gesto con cui si straccia ciò che abbiamo amato di più. Aracaeli è questo gesto], Il gioco segreto, op. cit., p. 199.

25. Voir Mengaldo P.V., "Vent'anni dopo La Storia. Omaggio a Elsa Morante», Studi Novecenteschi $\mathrm{n}^{\circ}$ 47-48, juin-décembre 1994.

26. On se souvient qu'interrogée sur Menzogna e sortilegio, E. Morante avait déclaré: «Io volevo scrivere l'ultimo romanzo possibile, l'ultimo romanzo della terra, naturalmente, anche il mio ultimo romanzo" (Il Mondo, 13 avril 1968).

27. Aracoeli, op. cit., p. 11. 